\title{
Image Denoising Based on Wavelet Transform using Visu Thresholding Technique
}

\author{
Pushpa Koranga, Garima Singh, Dikendra Verma \\ Department of Electronics and Communication Engineering \\ Graphic Era Hill University, Bhimtal Campus, Nainital, Uttarakhand, India \\ Shshank Chaube*, Anuj Kumar, Sangeeta Pant \\ Department of Mathematics \\ University of Petroleum \& Energy Studies, Dehradun, Uttarakhand, India \\ "Corresponding author: chaube.shshank@ gmail.com
}

(Received November 7, 2017; Accepted January 30, 2018)

\begin{abstract}
The image often contains noises due to several factors such as a problem in devices or due to an environmental problem etc. Noise is mainly undesired information, which degrades the quality of the picture. Therefore, image denoising method is adopted to remove the noises from the degraded image which in turn improve the quality of the image. In this paper, image denoising has been done by wavelet transform using Visu thresholding techniques for different wavelet families. PSNR (Peak signal to noise ratio) and RMSE (Root Mean Square Error) value is also calculated for different wavelet families.
\end{abstract}

Keywords- Wavelet transform, DWT, Hard threshold, Soft threshold.

\section{Introduction}

Image denoising is very important part of image processing which required in many fields such as Astrophysics, weather forecasting, medical etc. The main challenge is to recover original signal from the noisy image. In past, many methods have been adopted to denoise images. The discrete wavelet transform is widely used in image denoising due to the sparse representation of the image which means it has many coefficients close to zero (Wang et al., 2017). From the computational point of view, the discrete wavelet transform is very important (Ruikar and Doye, 2011). Earlier Fourier transform was used in image denoising but the signal was present in only frequency domain hence wavelet transform method was adopted which represent the signal in both frequency as well as time domain (Saluja and Boyat, 2015). Wavelet transform has very good energy compaction. The wavelet transform is applied to degraded image then soft or hard threshold method is applied. At last, the inverse wavelet transform is applied to obtain a reconstructed image (Om and Bishwas, 2015). The Wavelet threshold method denoised image by removing some insignificant coefficients with respect to some threshold value (Kumar and Saini, 2012). Donho and Johnstone proposed wavelet shrinkage method such as hard and soft thresholding for image denoising (Hedaoo and Godbole, 2011).

\section{Discrete Wavelet Transform}

Decomposition of the image has been done by wavelet transform, which has a wide range of applications in many fields (Kumar et al., 2011, 2016). Decomposition of the image is divided into two group first is a large number of coefficients which contain image features while the second group contains a small number of coefficients which contain noise features (Jaiswal et al., 
International Journal of Mathematical, Engineering and Management Sciences

Vol. 3, No. 4, 444-449, 2018

https://dx.doi.org/10.33889/IJMEMS.2018.3.4-032

2014). By applying discrete wavelet transform image is decomposed into four subband level and this subband with labeled HL1, LH1, HH1 and LL1 are formed by the horizontal and vertical filter (Anutam and Rajni, 2014; Deivalakshmi and Palanisamy, 2016). To obtain further higher decomposition LL1 is decomposed into HH2, LH2, HL2 and LL2 (Fig. 1). The LH1 subband is obtained from low pass filter in the horizontal direction and high pass filter from vertical direction whereas LL1 subband is obtained from low pass filter from both horizontal as well as vertical direction. The discrete wavelet transform is a simple denoising algorithm, which consists of three simple steps (Neelima and Pasha, 2014) (Fig. 2).

The discrete wavelet transform is applied to decompose the noisy image to obtain wavelet coefficients.

$>$ Wavelet thresholding techniques such as Visu shrink is applied to wavelet coefficients.

$>$ At last, the inverse Wavelet transform is applied to obtain a reconstructed image.
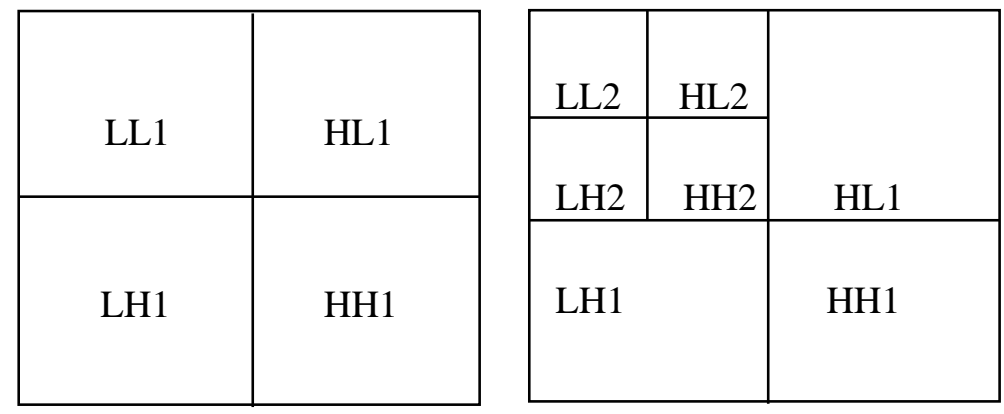

Fig. 1. Sub band for one level and two level of decomposition

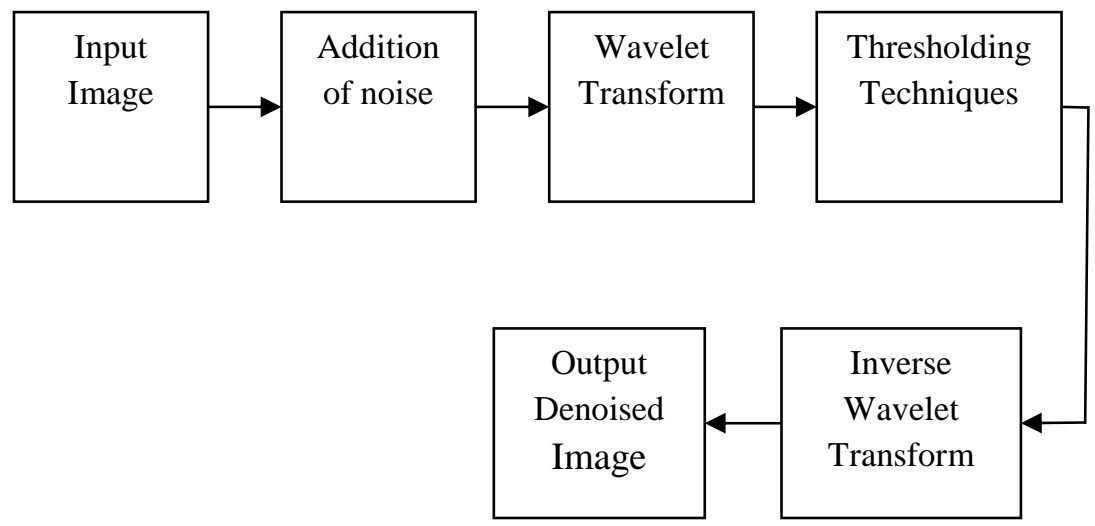

Fig. 2. Steps for wavelet transform 
International Journal of Mathematical, Engineering and Management Sciences

Vol. 3, No. 4, 444-449, 2018

https://dx.doi.org/10.33889/IJMEMS.2018.3.4-032

\section{Thresholding Techniques}

The thresholding technique is a simple non-linear method. In this technique, each wavelet coefficients is compared with the threshold value if all the coefficients value are smaller than the threshold value than it is set to zero otherwise it is set as it is or shrink all the coefficients value (Sudha et al., 2007; Tian et al., 2010). There are two types of thresholding techniques namely soft thresholding and hard thresholding.

\subsection{Soft Thresholding}

It is also called as Wavelet shrinkage function as it shrinks the wavelet coefficients towards the zero (Jhangra and Kumar, 2012). Soft thresholding produces visually pleasant image than hard thresholding (Saha et al., 2015). The soft thresholding function is given by (Naimi et al., 2015; Om and Biswas, 2012).

$$
\hat{\mathrm{W}}=\left\{\begin{array}{lr}
\mathrm{W}_{\mathrm{Y}}+\mathrm{T}, & \text { if } W_{Y} \geq T \\
W_{Y}-T, & \text { if } W_{Y} \leq T \\
0, & \text { if }\left|W_{Y}\right|<T
\end{array}\right.
$$

$\mathrm{W}_{\mathrm{y}}=$ Wavelet transform of noisy image

$\hat{\mathrm{W}}=$ Inverse wavelet transform of noisy image

$\mathrm{T}=$ Threshold value.

\subsection{Hard Thresholding}

The wavelet coefficient is set as it is, if larger than the threshold value otherwise set to zero (Liu, 2015). The hard threshold value is given by

$$
\hat{\mathrm{W}}= \begin{cases}\mathrm{W}_{\mathrm{Y}} & \text { when } \mathrm{W}_{\mathrm{Y}} \geq \mathrm{T} \\ 0 & \text { when } \mathrm{W}_{\mathrm{Y}}<T .\end{cases}
$$

\section{Visu Shrink}

Donho and Johnstone proposed Visu shrink thresholding technique, which is obtained by applying universal threshold (Rai et al., 2012; Gupta et al., 2013). The Visu shrink threshold is given by

$$
\mathrm{T}=\sigma \sqrt{2 \log N}
$$

where $\sigma=$ noise variance,

$\mathrm{N}=$ total number of pixel in the image.

The noise variance is given by

$$
\sigma=\frac{\operatorname{median}\left|\mathrm{HH}_{1}\right|}{0.6745}
$$

HH1 = Subband decomposition level.

Visu shrinks over smoothen the signal hence some detail or sharp edges of the images is lost (Biswas and Om, 2012). 
International Journal of Mathematical, Engineering and Management Sciences

Vol. 3, No. 4, 444-449, 2018

https://dx.doi.org/10.33889/IJMEMS.2018.3.4-032

\section{Results}

Table 1 gives the PSNR and RMSE value of different wavelet families for noise variance $\sigma=15$ using Visu thresholding technique for the second level of decomposition and denoised cameraman image using Visu thresholding technique for Db1 for the second level of decomposition is reflected in Fig. 3.

Table 1. PSNR and RMSE value of different wavelet families for noise variance $\sigma=15$ using Visu thresholding technique for the second level of decomposition

\begin{tabular}{|c|c|c|c|c|}
\hline Wavelet Families & PSNR (Hard) & PSNR (Soft) & RMSE (Hard) & RMSE (Soft) \\
\hline Haar & 15.5375 & 20.3161 & 42.6248 & 24.5887 \\
\hline Db1 & 15.6372 & 20.3977 & 42.1385 & 24.3588 \\
\hline Bior1.5 & 15.2568 & 20.1007 & 44.0252 & 25.2062 \\
\hline Sym1 & 15.5764 & 20.3396 & 42.4347 & 24.5223 \\
\hline Coif1 & 15.6081 & 20.5304 & 42.2801 & 23.9895 \\
\hline
\end{tabular}
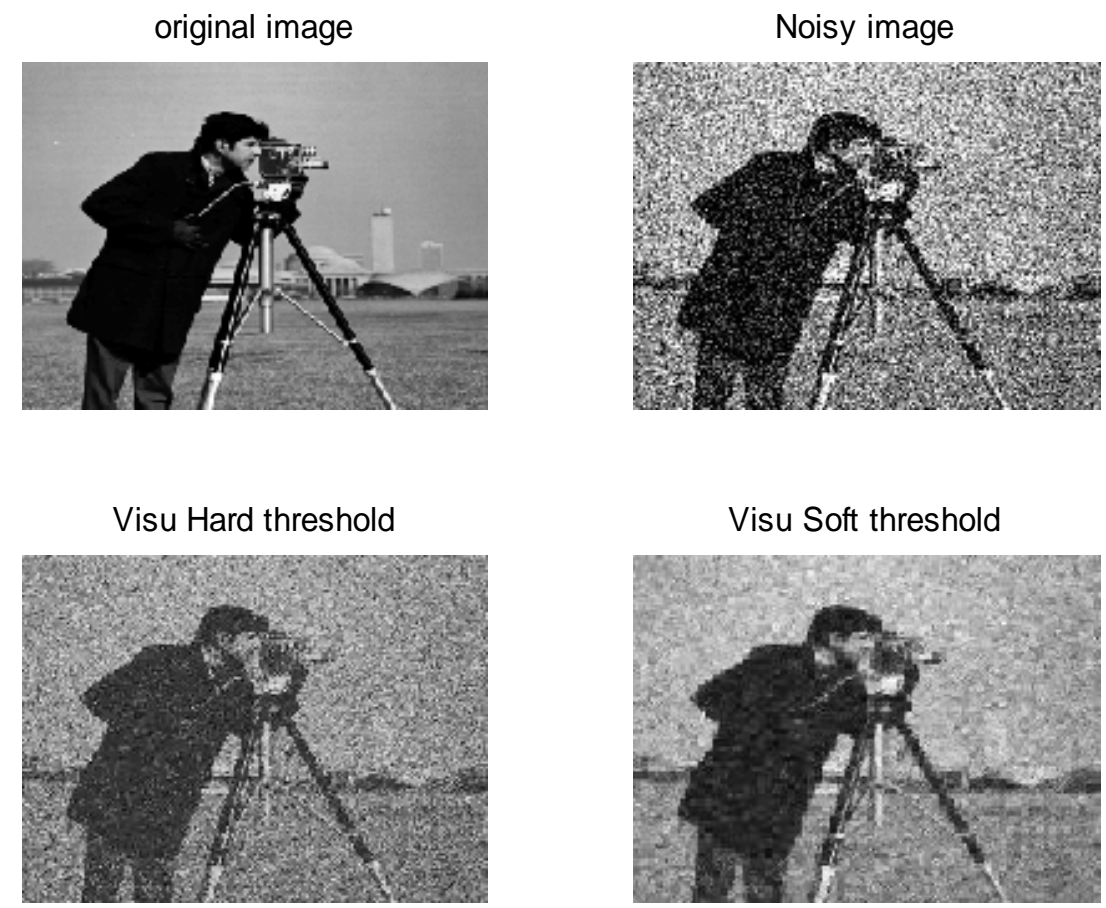

Fig. 3. Denoised cameraman image using Visu thresholding technique for $\mathrm{Db} 1$ for the second level of decomposition 
International Journal of Mathematical, Engineering and Management Sciences

Vol. 3, No. 4, 444-449, 2018

https://dx.doi.org/10.33889/IJMEMS.2018.3.4-032

\section{Conclusion}

In this paper cameraman image of $215^{*} 215$ size is used in tiff format. For cameraman image noise variance $\sigma=10$ is used. In this image Gaussian noise is used. The image is denoised by using both Visu soft and hard thresholding techniques. Peak Signal to Noise Ratio (PSNR) and Root Mean Square Error (RMSE) is calculated for different wavelet families such as Haar, Db1, Bior1.5, Sym1 and coif1 for the second level of decomposition.

\section{References}

Anutam, \& Rajni (2014). Performance analysis of image denoising with wavelet thresholding methods for different levels of decomposition. International Journal of Multimedia \& its Applications, 6(3), 35-46.

Biswas, M., \& Om, H. (2012). A new soft thresholding image denoising method. Procedia Technology, 6, 10-15.

Deivalakshmi, S., \& Palanisamy, P. (2016). Removal of high density salt and pepper noise through improved tolerance based selective arithmetic mean filtering with wavelet thresholding. International Journal of Electronics and Communication, 70(6), 757-776.

Gupta, V., Mahadeva, R., \& Shriwas, R. S. (2013, July). Image denoising using wavelet transform method. In Wireless and optical communications networks (wocn), 2013 tenth international conference on (pp. 1-4). IEEE.

Hedaoo, P., \& Godbole, S. S. (2011). Wavelet thresholding approach for image denoising. International Journal of Network Security and its Applications, 3(4), 16-21.

Jaiswal, A., Upadhyay, J., \& Somkuwar, A. (2014). Image denoising and quality measurements by using filtering and wavelet based techniques. International Journal of Electronics and Communication. 68(8), 699-705.

Jhangra, S., \& Kumar, S. (2012). A new threshold function for image denoising based on wavelet transform. International Journal of Engineering and Mathematical Sciences, 1(1), 60-65.

Kumar, A., Joshi, L. K., Pal, A. K., \& Shukla, A. K. (2011). MODWT based time scale decomposition analysis of BSE and NSE indexes financial time series. International Journal of Mathematical Analysis, 5(27), 1343-1352.

Kumar, A., Pant, S., \& Joshi, L. K. (2016). Wavelet variance, covariance and correlation analysis of BSE and NSE indexes financial time series. International Journal of Mathematical, Engineering and Management Sciences, 1(1), 26-33.

Kumar, R., \& Saini, B. S. (2012). Improved image denoising technique using neighboring coefficients of optimal wavelet with adaptive thresholding. International Journal of Computer Theory and Engineering, 4(3), 395-400.

Liu, Y. (2015). Image denoising method based on threshold, wavelet transform and genetic algorithm. International Journal of Signal Processing, Image processing and Pattern Recognition, 8(2), 29-40.

Naimi, H., Adamou-Mitiche, A. B. H., \& Mitiche, L. (2015). Medical image denoising using dual tree complex thresholding wavelet transform and wiener filter. Journal of King Saud University-Computer and Information Sciences, 27(1), 40-45. 
International Journal of Mathematical, Engineering and Management Sciences

Vol. 3, No. 4, 444-449, 2018

https://dx.doi.org/10.33889/IJMEMS.2018.3.4-032

Neelima, M., \& Pasha, M. M. (2014). Wavelet transform based on image denoising using thresholding techniques. International Journal of Advanced Research in Computer and Communication Engineering, 3(9), 7906-7908.

Om, H., \& Bishwas, M. (2015). A generalized image denoising method using neighbouring wavelet coefficients. Signal, Image and Video Processing, 9(1), 191-200.

Om, H., \& Biswas, M. (2012). A new image denoising scheme using soft-thresholding. Journal of Signal and Information Processing, 3, 360-363.

Rai, R. K., Asnani, J., \& Sontakke, T. R (2012). Review of shrinkage techniques for image denoising for image denoising. International Journal of Computer Applications, 42(19), 13-16.

Ruikar, S. D., \& Doye, D. D. (2011). Wavelet based image denoising technique. International Journal of Advanced Computer Science and Applications, 2(3), 49-53.

Saha, M., Naskar, M. K., \& Chatterji, B. N. (2015). Soft, hard and block thresholding techniques for denoising of mammogram images. IETE Journal of Research, 61(2), 1-6.

Saluja, R., \& Boyat, A. (2015). Wavelet based image denoising using weighted highpass filtering coefficients and adaptive weiner filter. IEEE International Conference on Computer and Control (IC42015).

Sudha, S., Suresh, G. R., \& Sukanesh, R. (2007, December). Wavelet based image denoising using adaptive thresholding. In Conference on Computational Intelligence and Multimedia Applications, 2007. International Conference on (Vol. 3, pp. 296-300). IEEE.

Tian, M., Wen, H., Zhou, L., \& You, X. (2010, July). Image denoising using multi-scale thresholds method in the wavelet domain. In Wavelet Analysis and Pattern Recognition (ICWAPR), 2010 International Conference on (pp. 79-83). IEEE.

Wang, J., Wu, J., Wu, Z., Jeong, J., \& Jeon, G. (2017). Wiener filter-based wavelet domain denoising. Displays, 46, 37-41. 